\title{
TIGAR promotes neural stem cell differentiation through acetyl-CoA- mediated histone acetylation
}

Wenjuan Zhou', Tiantian Zhao ${ }^{1}$, Jingyi Du', Guangyu $\mathrm{Ji}^{1}$, Xinyue $\mathrm{Li}^{1}$, Shufang $\mathrm{Ji}^{1}$, Wenyu Tian $\mathbb{D}^{1}$, Xu Wang ${ }^{1}$ and Aijun $\mathrm{HaO}^{1}$

\begin{abstract}
Cellular metabolism plays a crucial role in controlling the proliferation, differentiation, and quiescence of neural stem cells (NSCs). The metabolic transition from aerobic glycolysis to oxidative phosphorylation has been regarded as a hallmark of neuronal differentiation. Understanding what triggers metabolism reprogramming and how glucose metabolism directs NSC differentiation may provide new insight into the regenerative potential of the brain. TP53 inducible glycolysis and apoptosis regulator (TIGAR) is an endogenous inhibitor of glycolysis and is highly expressed in mature neurons. However, its function in embryonic NSCs has not yet been explored. In this study, we aimed to investigate the precise roles of TIGAR in NSCs and the possible involvement of metabolic reprogramming in the TIGAR regulatory network. We observed that TIGAR is significantly increased during brain development as neural differentiation proceeds, especially at the peak of NSC differentiation (E14.5-E16.5). In cultured NSCs, knockdown of TIGAR reduced the expression of microtubule-associated protein 2 (MAP2), neuron-specific class III beta-tubulin (Tuj1), glial fibrillary acidic protein (GFAP), Ngn1, and NeuroD1, and enhanced the expression of REST, suggesting that TIGAR is an important regulator of NSC differentiation. Furthermore, TIGAR enhanced the expression of lactate dehydrogenase $\mathrm{B}(\mathrm{LDHB})$ and the mitochondrial biogenesis and oxidative phosphorylation (OXPHOS) markers, peroxisome proliferator-activated receptor gamma coactivator 1 (PGC-1a), nuclear respiratory factor (NRF1), and MitoNEET during NSC differentiation. TIGAR can decrease lactate production and accelerate oxygen consumption and ATP generation to maintain a high rate of OXPHOS in differentiated NSCs. Interestingly, knockdown of TIGAR decreased the level of acetyl-CoA and H3K9 acetylation at the promoters of Ngn1, Neurod1, and Gfap. Acetate, a precursor of acetyl-CoA, increased the level of H3K9 acetylation and rescued the effect of TIGAR deficiency on NSC differentiation. Together, our data demonstrated that TIGAR promotes metabolic reprogramming and regulates NSC differentiation through an epigenetic mechanism.
\end{abstract}

\section{Introduction}

NSCs are defined by their capacity for self-renewal and for differentiation into neurons, astrocytes, and oligodendrocytes ${ }^{1}$. NSCs can generate new neurons in the

\footnotetext{
Correspondence: Aijun Hao (aijunhao@sdu.edu.cn)

${ }^{1}$ Key Laboratory of the Ministry of Education for Experimental Teratology, Shandong Provincial Key Laboratory of Mental Disorders, Department of Human Anatomy and Histoembryology, School of Basic Medical Sciences, Shandong University, Jinan, Shandong, China

Edited by S. Tait
}

course of normal physiological activity in both developing and adult mammalian brains to guarantee the integrity and precision of neural connection and cerebral function. However, in most neurodegenerative diseases and most brain trauma, neuronal damage is irreversible; the neuron population cannot be refreshed through NSC differentiation. A full understanding of the differentiation potential of NSCs may help us apply cell-replacement therapeutic strategies against neurological diseases ${ }^{2,3}$. Growing evidence has shown that multiple metabolic

\section{(c) The Author(s) 2019}

(c) (i) Open Access This article is licensed under a Creative Commons Attribution 4.0 International License, which permits use, sharing, adaptation, distribution and reproduction in any medium or format, as long as you give appropriate credit to the original author(s) and the source, provide a link to the Creative Commons license, and indicate if changes were made. The images or other third party material in this article are included in the article's Creative Commons license, unless indicated otherwise in a credit line to the material. If material is not included in the article's Creative Commons license and your intended use is not permitted by statutory regulation or exceeds the permitted use, you will need to obtain permission directly from the copyright holder. To view a copy of this license, visit http://creativecommons.org/licenses/by/4.0/. 
pathways participate in cell fate determination in $\mathrm{NSCs}^{4}$. Metabolic dysfunction is closely associated with many neurological diseases, including schizophrenia as well as Alzheimer's (AD), Parkinson's (PD), and Huntington's diseases $(\mathrm{HD})^{5}$. Given the important role of metabolism in regulating the proliferation and differentiation of NSCs, pharmacological intervention in metabolic homeostasis may be able to exploit the regenerative potential of NSCs against these neurodegenerative diseases.

TIGAR is generally regarded as an antiapoptotic gene expressed in response to p53-induced cell death. As a bisphosphatase, TIGAR reduces intracellular fructose-2,6bisphosphate levels, resulting in an inhibition of glycolysis ${ }^{6}$. TIGAR is highly expressed in human breast carcinoma cells. Recent evidence showed that overexpression of TIGAR in carcinoma cells altered metabolic compartmentalization to a mitochondrial metabolic phenotype and ultimately increased tumor growth ${ }^{7}$. Importantly, TIGAR is widely distributed in neurons and plays crucial roles in the central nervous system (CNS). For example, in ischemic stroke, TIGAR protects against ischemic/reperfusion-induced injury via glucose 6phosphate dehydrogenase-enhanced pentose phosphate pathway (PPP) flux ${ }^{8}$. However, in the developing brain, the effect of TIGAR in NSCs is largely unknown.

Glucose is the obligatory energy substrate in the brain. The regulation of glucose metabolism in the CNS is a key process that has been investigated for many years ${ }^{9,10}$. Studies have confirmed that NSCs rely mainly on aerobic glycolysis to maintain their energy supply, while mature neurons depend on oxidative phosphorylation ${ }^{4}$. Reduced levels of oxygen enhance the survival and proliferation of cultured NSCs and inhibit differentiation. NSCs also display higher lactate dehydrogenase activity, more lactate production, and higher glucose consumption than cultured neurons ${ }^{11}$. Importantly, metabolic reprogramming from aerobic glycolysis to oxidative phosphorylation (OXPHOS) has been found during neuronal differentiation. Decreased expression of hexokinase (HK2) and lactate dehydrogenase A (LDHA), and a switch in pyruvate kinase gene splicing from PKM2 to PKM1 were found during NSC differentiation ${ }^{12}$. However, it remains unclear what conditions can trigger metabolic transition and how metabolism regulates neuronal differentiation. It is well known that OXPHOS can supply more energy than glycolysis during neuronal differentiation to enable protein synthesis and neuronal activity. The role of metabolites produced during glucose metabolism cannot be ignored during cell fate decisions of NSCs.

As mentioned, TIGAR is an important endogenous inhibitor of glycolysis. Whether TIGAR participates in metabolic reprogramming during NSC differentiation is unknown. In this study, we explored the effect of TIGAR in NSCs and investigated the potential molecular mechanism of TIGAR-induced NSC differentiation.
Fundamental understanding of the metabolic mechanisms of TIGAR in NSCs may help provide new therapies that reactivate neurogenesis to treat degenerative diseases.

\section{Material and methods \\ Primary NSC culture}

Primary NSCs were isolated and cultured as previously described $^{13,14}$. Briefly, brains were obtained from Kun Ming mice at embryonic day 12.5 (E12.5). Then, the brains were cut mechanically and digested with $0.25 \%$ trypsin (Gibco, USA) for $5 \mathrm{~min}$ at $37^{\circ} \mathrm{C}$. Dulbecco's modified Eagle's medium (DMEM)/F12 (1:1) medium (Gibco, USA) was used to neutralize the effect of trypsin. After centrifugation, NSCs were resuspended and $2 \times 10^{5} / \mathrm{ml}$ cells were seeded in $25-\mathrm{cm}^{2} \mathrm{~T}$-flasks with DMEM/F12 medium supplemented with $2 \%$ B27 (Gibco, USA), $20 \mathrm{ng} / \mathrm{ml} \mathrm{bFGF}$ (R\&D, USA), $20 \mathrm{ng} / \mathrm{ml}$ epidermal grown factor (EGF; Invitrogen, USA), and $1 \%$ penicillin/streptomycin solution. Cells were incubated at $37^{\circ} \mathrm{C}$ under a $5 \% \mathrm{CO}_{2}$ atmosphere for five days. Then, the primary neurospheres were collected and digested using trypsin and EDTA, and incubated in serum-free medium for another $3-5$ days (passage 1 neurospheres). To investigate NSC differentiation, we dissociated passage 1 neurospheres, stimulated them with differentiation medium containing $2 \%$ fetal bovine serum (Gibco, USA) without growth factors, and cultured them for 1-9 days. All animal experiments complied with the National Institutes of Health Guide for the Care and Use of Laboratory Animals and were approved by the Institutional Animal Care and Use Committees of Shandong University (no. 201402020).

\section{RNA interference plasmid constructs and lentivirus packaging}

Plasmid constructs were designed as previously described $^{15}$. The Tigar shRNA forward sequences 5'-TTA GCA GCC AGC ATC TTA GTT CAA GAG ACT AAG ATG CTG GCT GCT AAT TTT TT-3' and reverse sequences 5'-AAT TAA AAA ATT AGC AGC CAG CAT CTT AGT CTC TTG AAC TAA GAT GCT GGC TGC TAA GGC C-3' were annealed and ligated into pSilencer 1.0 vector. Then the U6 promoter and the Tigar shRNA sequences were cut from pSilencer 1.0 and inserted into PGW vector. The PGW plasmid was a lentiviral transfer vector and contained a Gfp reporter gene. The pUltra plasmid was used to package Tigar or Tigar-TM mutant lentivirus. In addition, three package plasmids pMDL/ pRRE, VSV-G, and pRSV-REV were used in the experiments. To produce high titer lentiviruses, these above plasmids were transfected into $293 \mathrm{~T}$ cells.

\section{RNA isolation and real-time quantitative PCR}

TRIZOL reagent (Invitrogen, Carlsbad, CA, USA) was used to isolate total RNA from cultured NSCs. The purity 
Table 1 primer sequence

\begin{tabular}{|c|c|c|}
\hline Gene & Forward primer & Reverse primer \\
\hline Tigar & AGGGCAGAGAGAAAGCGT & TGCCACCTITGGGATTC \\
\hline Nestin & CCACAGTGCCCAGTTCTA & ATAGAGTGGTGAGGGTTGAG \\
\hline Sox2 & ACTCCATGACCAGCTCGCAGAC & CCTCGGACTTGACCACAG \\
\hline Gfap & AACAACCTGGCTGCGTAT & ACTGCCTCGTATTGAGTGC \\
\hline Map2 & GAATAAGCAAGAGCCCAGAG & GTCCGTCGTGCTGAAGAG \\
\hline Neurod1 & CTCAGCATCAATGGCAACTTC & CACCGGAAGAGAAGATTGATC \\
\hline Rest & CATGGCCTTAACCAACGACAT & CGACCAGGTAATCGCAGCAG \\
\hline Ngn 1 & AGTAGTCCCTCGGCTTCAGA & ATGAAACAGGGCGTCGTG \\
\hline Ki67 & AAGAGTGAGGGAATGCCTAT & TCATTTGTCCTCGGTGGC \\
\hline Glut1 & CTGGGAATCGTCGTTGG & GCAGAAGGGCAACAGGATA \\
\hline Glut3 & GCTGGGCATCGTTGTTG & CTGTAGCTTGGTCTTCCTCCT \\
\hline Mct1 & GAGGTCCTATCAGCAGTATCTT & CCAGTGGTCGCTTCTTGT \\
\hline Mct2 & CACTGGCTCCTITCAATC & CTGGCTTTCTTCAGAGTTG \\
\hline Mct4 & ACTTCAACAAGCGTCGCCCTAT & TCAGTCCCTCCGCCTACCTG \\
\hline Pfkfb3 & AGCCTCTTGACCCTGATA & TTCTTGCCTCTGCTGGAC \\
\hline Ldha & GTTGGGGTTGGTGCTGTT & ATCTCGCCCTTGAGTTTG \\
\hline Ldhb & TGGACAAGTGGGTATGGC & TTाCGGAGTCTGGAGGA \\
\hline Pgc-1a & TGGAGCAATAAAGCGAAGA & GCGGTTGTGTATGGGACT \\
\hline Nrf1 & TCCCAGAGATGCTCAAGTA & CTATGGTCCGTAATGCCT \\
\hline Mitoneet & AGAGAATCGCACCAAAGC & GTCGCCAGTCTCTTCGTT \\
\hline Tomm20 & AGCACAAGGTGACTACGAG & TGACTAATGGTCGGAAGC \\
\hline Acly & ACGTGTGCATCTATGCTAC & GGACCAACAGGTGTCTCTTA \\
\hline Acss2 & СCTCTACTGCTITGTTACCT & GAGCGTGTITAGGGAAG \\
\hline$\beta$-actin & CGTTGACATCCGTAAAGACCTC & CCACCGATCCACACAGAGTAC \\
\hline Ngn1 (ChIP) & CATTGTTGCGCGCCGTA & GCGATCAGATCAGCTCCT \\
\hline Neurodl (ChIP) & GTCCGCGGAGTCTCTAACTG & GAACCACGTGACCTGCCTAT \\
\hline Ngn1 (ChIP) & ACAAAAGGCCTGGGTTGACA & CTCTGGATCTGGAACTCGCC \\
\hline
\end{tabular}

and concentration of total RNA was measured by a spectrophotometer. Then cDNA were synthesized with a RevertAid $^{\mathrm{TM}}$ First Strand cDNA Synthesis Kit (Thermo Fisher Scientific). Real-time PCR was performed with SYBR Green Realtime PCR Master Mix (TOYOBO CO., Ltd., Japan). The expression of $\beta$-actin was regarded as a normalization control, and the $2^{-\Delta \Delta C T}$ method was used to calculate changes of the gene expression levels. The primer sequences were listed in Table 1.

\section{Immunofluorescence}

Cultured NSCs or embryonic brain sections were fixed with $4 \%$ paraformaldehyde for $30 \mathrm{~min}$ and rinsed with PBS (pH 7.4) three times. Cells or brain sections were blocked with $10 \%$ goat serum containing $0.3 \%$ Triton
$\mathrm{X}-100$ for $2 \mathrm{~h}$ and then incubated with primary antibodies overnight at $4{ }^{\circ} \mathrm{C}$. The following primary antibodies were used: rabbit anti-TIGAR (1:500, ab37910; Abcam), mouse anti-Nestin (1:200, ab6142; Abcam), mouse anti-Sox2 (1:200, ab171380; Abcam), mouse anti-Tuj1 (1:200, \#4466; CST), mouse anti-GFAP (1:200, BA0056; Boster), and rabbit anti-GFP (1:200, \#2956; CST). After being washed three times in PBS, the cells or slices were incubated with secondary antibodies conjugated to FITC or TRITC for $1 \mathrm{~h}$ at room temperature. The sections were stained with $2 \mu \mathrm{g} / \mathrm{ml}$ 4',6-diamidino-2- phenylindole (DAPI) before being photographed. Images were obtained with an IX71 Olympus fluorescence microscope. 5-Ethynyl-2'-deoxyuridine (EdU) labeling assays were detected using a Cell-Light EdU Apollo 488 In Vitro kit (Ribobio, 
Guangzhou, China). NSCs cultured in differentiation medium for 5 days were treated with $50 \mu \mathrm{M}$ EdU for $2 \mathrm{~h}$ according to the manufacturer's protocol. Then, cultured NSCs were fixed with $4 \%$ paraformaldehyde for EdU staining. The number of EdU- or DAPI-positive cells was counted with Image). TUNEL staining was performed using the Fluorescein In Situ Apoptosis Detection Kit (KeyGEN BioTECH, China).

\section{Western blotting}

In vitro cultured NSCs or mouse embryonic cortical tissue was homogenized in RIPA buffer (Beyotime Institute of Biotechnology, Shanghai, China) containing protease and phosphatase inhibitors for $30 \mathrm{~min}$ at $4^{\circ} \mathrm{C}$. After centrifugation at $12000 \mathrm{rpm}$ at $4{ }^{\circ} \mathrm{C}$ for $15 \mathrm{~min}$, the supernatants were collected. The protein concentration was measured using the Pierce bicinchoninic acid (BCA) assay kit. The samples were mixed with $5 \times$ loading buffer (Boster, Wuhan, China), and equal amounts of protein were loaded into each well of $8-15 \%$ SDS-PAGE gels. Then, the separated proteins were transferred to PVDF membranes (BioRad). After being blocked with 5\% milk in TBST buffer for $2 \mathrm{~h}$, the membranes were incubated with primary antibodies at $4{ }^{\circ} \mathrm{C}$ overnight and then with HRP conjugated secondary antibodies for $1 \mathrm{~h}$ at RT. After being washed with TBST buffer, blots were visualized using an enhanced chemiluminescence (ECL) kit (Merck Millipore). Primary antibodies were used at the following dilutions: rabbit anti-TIGAR (1:1000, ab37910; Abcam), rabbit anti- $\beta$-actin (1:2000, \#4970; CST), mouse anti-Tuj1 (1:1000, \#4466; CST), mouse anti-GFAP (1:1000, BA0056; Boster), mouse anti-GLUT1 (1:500, sc-377228; Santa), mouse anti-MCT1 (1:500, sc365501; Santa), mouse anti-LDHA (1:500, sc-137243; Santa), mouse anti-LDHB (1:500, sc-100775; Santa), rabbit anti-H3K9ac (1:1000, \#9649; CST), rabbit anti-H3K18ac (1:1000, \#9675; CST), rabbit anti-H3K14ac (1:1000, \#7627; CST), rabbit anti-H3K27ac (1:1000, \#4353; CST), and rabbit anti-H3 (1:1000, \#4499; CST).

\section{Mitochondrial isolation and acetyl-CoA quantification}

A mitochondria isolation kit (ab110170, Abcam) was used in this experiment ${ }^{16,17}$. In brief, cultured NSCs were frozen and thawed to weaken the membranes and suspended in Reagent A. Then, the cells were homogenized and centrifuged at $1000 \times g$ at $4{ }^{\circ} \mathrm{C}$ for $10 \mathrm{~min}$. The supernatant was collected (SN1), and the pellet was resuspended with Reagent B. After homogenization and centrifugation, the supernatant was collected again (SN2). SN1 and SN2 were mixed thoroughly and centrifuged at $12,000 \times g$ for $15 \mathrm{~min}$ at $4{ }^{\circ} \mathrm{C}$. The pellet was collected in Reagent $\mathrm{C}$ supplemented with protease inhibitors and used for acetyl-CoA quantification.

Acetyl-CoA quantification was conducted using an acetyl-CoA assay kit (Solarbio life sciences, Beijing,
China). Briefly, $5 \times 10^{6}$ cells or isolated mitochondria were collected and incubated in extraction buffer for $30 \mathrm{~min}$. The cells were subjected to sonication and centrifuged at $8000 \times g$ at $4{ }^{\circ} \mathrm{C}$ for $10 \mathrm{~min}$. The supernatants were collected and supplemented with acetyl-CoA assay buffer. The $340 \mathrm{~nm}$ absorbance values were measured at $20 \mathrm{~s}$ $\left(\mathrm{A}_{20 \mathrm{~s}}\right)$ and $80 \mathrm{~s}\left(\mathrm{~A}_{80 \mathrm{~s}}\right)$. The difference between $\mathrm{A}_{80 \mathrm{~s}}$ and $A_{20 S}$ was used to calculate the relative level of acetyl-CoA.

\section{Fluorescent glucose uptake assay, lactate production assay, and ATP measurement}

Cultured cells were rinsed with Krebs-Ringer-HEPES (KRH) buffer and incubated for $30 \mathrm{~min}$ in the presence of $600 \mu \mathrm{M}$ 2-[N-(7-nitrobenz-2-oxa-1,3-diazol-4-yl)amino] -2-deoxyglucose (2-NBDG), a fluorescent glucose tracer used to measure glucose transport ${ }^{18,19}$. Then, the cells were washed with PBS to remove the unabsorbed 2-NBDG and fixed with $4 \%$ paraformaldehyde. The accumulation of 2-NBDG in cells was imaged by a fluorescence microscopy. NIH Image J software was used to calculate the fluorescence value of each group. Ten non-overlapping images were analyzed in each group.

Lactate release was measured using a lactate assay kit (1200011002, Eton Bioscience), and ATP was measured using a luminescent ATP detection assay kit (ab113849, Abcam) according to the manufacturer's protocol.

\section{Oxygen consumption rate assay}

For analysis of mitochondrial respiratory potential, Seahorse Bioscience XFe 96 Extracellular Flux Analyzer was used to monitor Oxygen consumption rate (OCR) in differentiated NSCs according to the manufacturer's instructions. $8 \times 10^{4}$ cells per well were seeded on PDLcoated XFe 96 plates, and incubated at $37^{\circ} \mathrm{C}$ in $5 \% \mathrm{CO}_{2}$. The NSCs were transfected with Lenti-Gfp or Lenti-Tigar for $72 \mathrm{~h}$. On the day of detection, the cells were washed with XF basal medium containing $17.5 \mathrm{mM}$ glucose and $1 \mathrm{mM}$ sodium pyruvate $(\mathrm{pH} 7.4)$ and incubated at $37^{\circ} \mathrm{C}$ in a non- $\mathrm{CO}_{2}$ incubator for $1 \mathrm{~h}$. OCR was measured with sequential injections of $1 \mu \mathrm{M}$ oligomycin, $0.5 \mu \mathrm{M}$ FCCP and $0.5 \mu \mathrm{M}$ each of rotenone/antimycin A. OCR was normalized to the protein concentration in each well.

\section{Chromatin immunoprecipitation assay}

Chromatin immunoprecipitation (ChIP) was conducted using an EZ-ChIP kit (Merck Millipore). Cultured NSCs were treated with $1 \%$ formaldehyde for $15 \mathrm{~min}$ at RT to generate cross-links between histones and DNA. Then, the cells were washed three times with PBS and collected in SDS lysis buffer supplemented with protease inhibitors. After sonication, the chromatin in each cell lysate sample was sheared into 200-500 bp fragments. The supernatants were immunoprecipitated with a specific antibody (antiH3K9ac, CST) or a control antibody (anti-IgG) overnight 
at $4{ }^{\circ} \mathrm{C}$. Then, the supernatants were subjected to washing, elution and cross-link reversal following the manufacturer's instructions. Purified genomic DNA from the supernatants was analyzed using real-time PCR. The primers were listed in Table 1.

\section{Statistical analysis}

Statistical analysis was performed using SPSS program (version 19.0). Data statistical significance was calculated by Student's $t$-test or one-way or two-way repeated ANOVA, followed by the LSD or Dunnett's T3 post hoc test. Values were presented as the mean \pm SEM and significance was set at $p<0.05$.

\section{Results}

TIGAR is highly expressed in the embryonic neocortex and NSCs

To investigate the role of TIGAR in brain development, we assessed the expression pattern of TIGAR from E10.5 to E18.5 during embryonic cortex development. Both qRT-PCR and western blot results showed that the expression of TIGAR was significantly increased during cortical development, especially during the peak of NSC differentiation (E14.5 and E16.5) (Fig. 1a, b). Similar results were obtained in immunostaining experiments, and TIGAR was widely distributed in the embryonic cortex, as indicated by its localization in the ventricular zone (VZ), the subventricular zone (SVZ) and the cortical plate (CP) at E16.5 (Fig. 1c, d). Nestin and Sox2 are two markers of NSCs. By immunofluorescent staining for TIGAR and Nestin or Sox2, we found that TIGAR is localized in the cytoplasm of NSCs (Fig. 1e, f). Studies have demonstrated that the time from E12.5 to E16.5 is an important period for the initiation of neuronal differentiation $^{20}$. The data showing that the expression levels of TIGAR increased over the course of neural differentiation suggested that TIGAR may play crucial roles in neural differentiation during embryonic cortex development.

To evaluate whether TIGAR is involved in the differentiation of NSCs, we further analyzed the expression of TIGAR during the proliferation and differentiation of cultured NSCs. NSCs were isolated and incubated with different media. In line with the expression of Map2 (a neuronal marker) and Gfap (an astrocyte marker), Tigar was especially increased during the differentiation of NSCs and, conversely, decreased during proliferation (Fig. 2). These data suggest that TIGAR may be an important regulator of NSC differentiation during embryonic development.

\section{TIGAR is necessary and sufficient for NSC differentiation}

To further analyze the effect of TIGAR in NSCs, we used modified lentiviruses containing specific sequences to knock down or overexpress Tigar. As shown in Fig. 3a, knockdown of TIGAR significantly decreased the mRNA levels of Map2 and Gfap in the differentiation process. It has been reported that the transcriptional activator factors Ngn1 and Neurod1 and the repressor factor Rest are required for the differentiation of $\mathrm{NSCs}^{21,22}$. We found that loss of TIGAR significantly decreased the expression levels of Ngn1 and Neurod1 and increased the expression of Rest (Fig. 3a). Western blots also revealed a decrease in Tuj1 (a neuronal marker) and GFAP (Fig. 3b, c). These results suggest that TIGAR is necessary for NSC differentiation. In addition, overexpression of TIGAR increased the expression of MAP2, GFAP, Tuj1, NeuroD1, and Ngn1, which suggests that TIGAR is sufficient for NSC differentiation (Fig. 3d-f). In our immunofluorescent staining experiments, GFP-positive cells represent lentivirus-infected NSCs. We found that after stimulation with Lenti-siTigar the ratio of Tuj $1^{+} \mathrm{GFP}^{+}$to $\mathrm{GFP}^{+}$cells was significantly decreased (Fig. 3g). Lenti-siTigar also reduced the ratio of $\mathrm{GFAP}^{+} \mathrm{GFP}^{+}$to $\mathrm{GFP}^{+}$cells compared with the ratio in the Lenti-siSCR treated group (Fig. 3h). Our data revealed that TIGAR can regulate the differentiation of NSCs.

Under proliferation conditions, overexpression of TIGAR decreased the expression of Nestin and increased the expression of Gfap, but Map2 was unchanged, suggesting that the role of TIGAR in proliferation is slight and that TIGAR is more likely to accelerate NSC differentiation during differentiated conditions (Fig. 4a, b). We further examined the effect of TIGAR on cell survival and cell proliferation by TUNEL staining and an EdU incorporation assay, respectively, during the differentiation stage. TUNEL staining showed that knockdown of TIGAR had little effect on cell survival (Fig. 4c). The EdU incorporation assay showed that the percentage of EdU-positive cells was similar between the Lenti-siTigar and Lenti-siSCR treated groups (Fig. 4d). Collectively, our data demonstrate that TIGAR is necessary and sufficient for NSC differentiation.

\section{TIGAR promoted a shift in glucose metabolism to a mitochondrial phenotype and increased the levels of acetyl-CoA during NSC differentiation}

As mentioned above, TIGAR is an endogenous inhibitor of glycolysis. To investigate the effect of TIGAR on glycolysis in NSCs, we analyzed the expression levels of transporters and metabolic enzymes after knockdown of TIGAR. The results showed that the mRNA and protein expression levels of GLUTs, MCTs, PFKFB3, and LDHA were not changed after stimulation with Lenti-siTigar (Fig. 5a, b). However, knockdown of TIGAR decreased the expression of LDHB, which is an important subunit of lactate dehydrogenase that converts lactate into pyruvate for mitochondrial metabolism (Fig. 5a, b). 2-NBDG was used to the measure glucose uptake of NSCs. As shown in Fig. 5c, knockdown of TIGAR had no significant effect on 


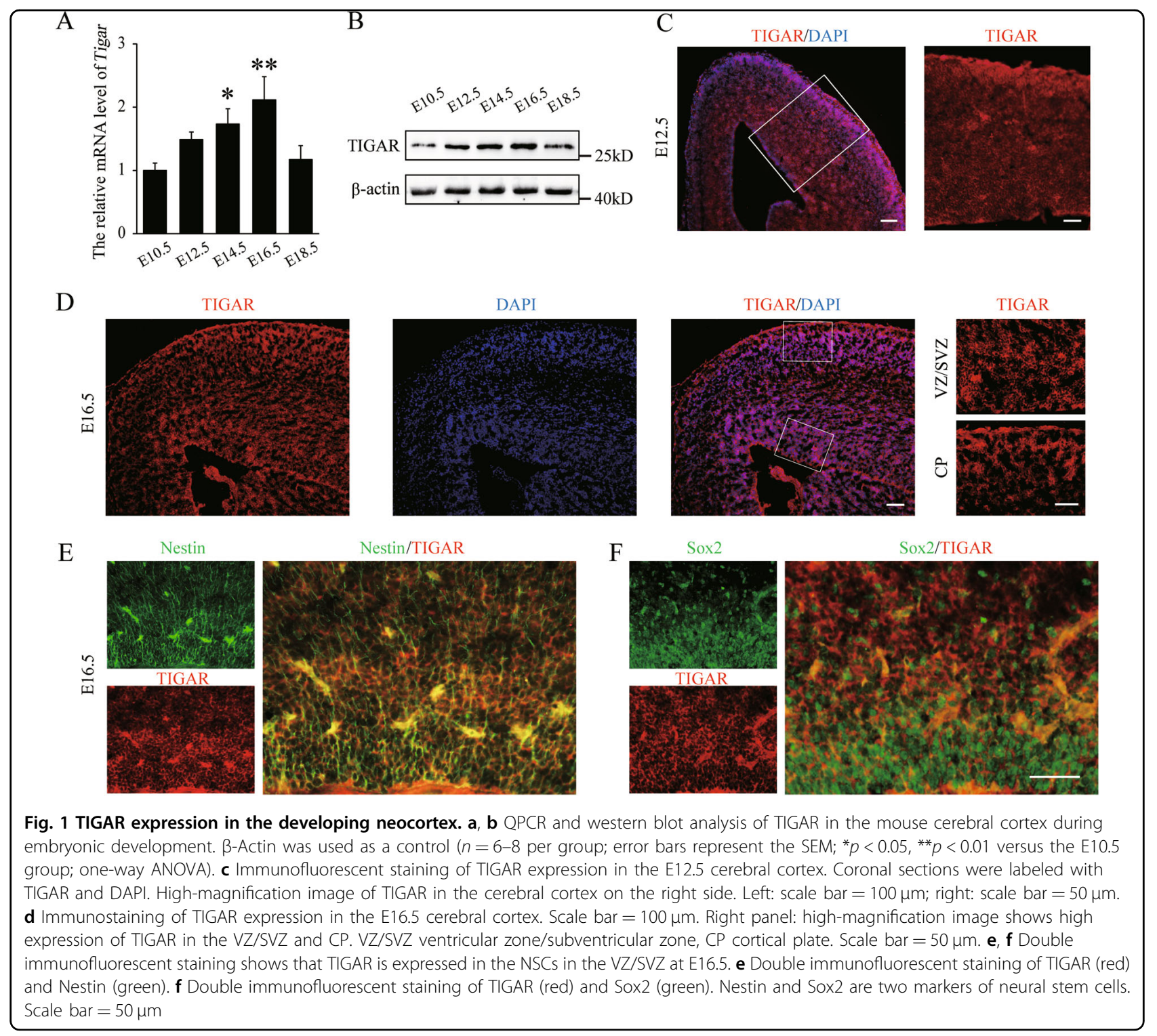

glucose uptake. As expected, the lactate production, as a measure of glycolysis, was increased after knockdown of TIGAR (Fig. 5d). In addition, we examined the expression of the mitochondrial biogenesis markers PGC- $1 \alpha$ and NRF1, and the OXPHOS markers MitoNEET and transporter of the outer mitochondrial membrane member 20 (Tomm20) in differentiated NSCs. We found that knockdown of TIGAR decreased the expression of $P g c-1 \alpha$, $\mathrm{Nrf1}$, and Mitoneet, while overexpression of TIGAR increased the expression of these genes (Fig. 5e, f). To further verify the effect of TIGAR on OXPHOS, oxygen consumption and ATP generation were detected in NSCs. Compared with Lenti-Gfp-treated group, overexpression of TIGAR increased the oxygen consumption rate in differentiated NSCs (Fig. 5g). Besides, as shown in Fig. 5h, knockdown of TIGAR significantly decreased the level of ATP. These results suggest that TIGAR may enhance the transition from lactate to pyruvate and promote OXPHOS during NSC differentiation.

Evidence has demonstrated that metabolic reprogramming can induce alteration of cellular acetyl-CoA levels ${ }^{23,24}$. Interestingly, we found that the level of acetyl-CoA in total cell lysate was significantly decreased after TIGAR deficiency (Fig. 5i). After isolation of mitochondria, our data further revealed that knockdown of TIGAR reduced the production of acetyl-CoA in mitochondria (Fig. 5i). After knockdown of TIGAR, the decrease in LDHB expression and mitochondrial activity may cause less pyruvate to become acetyl-CoA in mitochondria. Acetate-dependent acetyl-CoA synthetase 2 


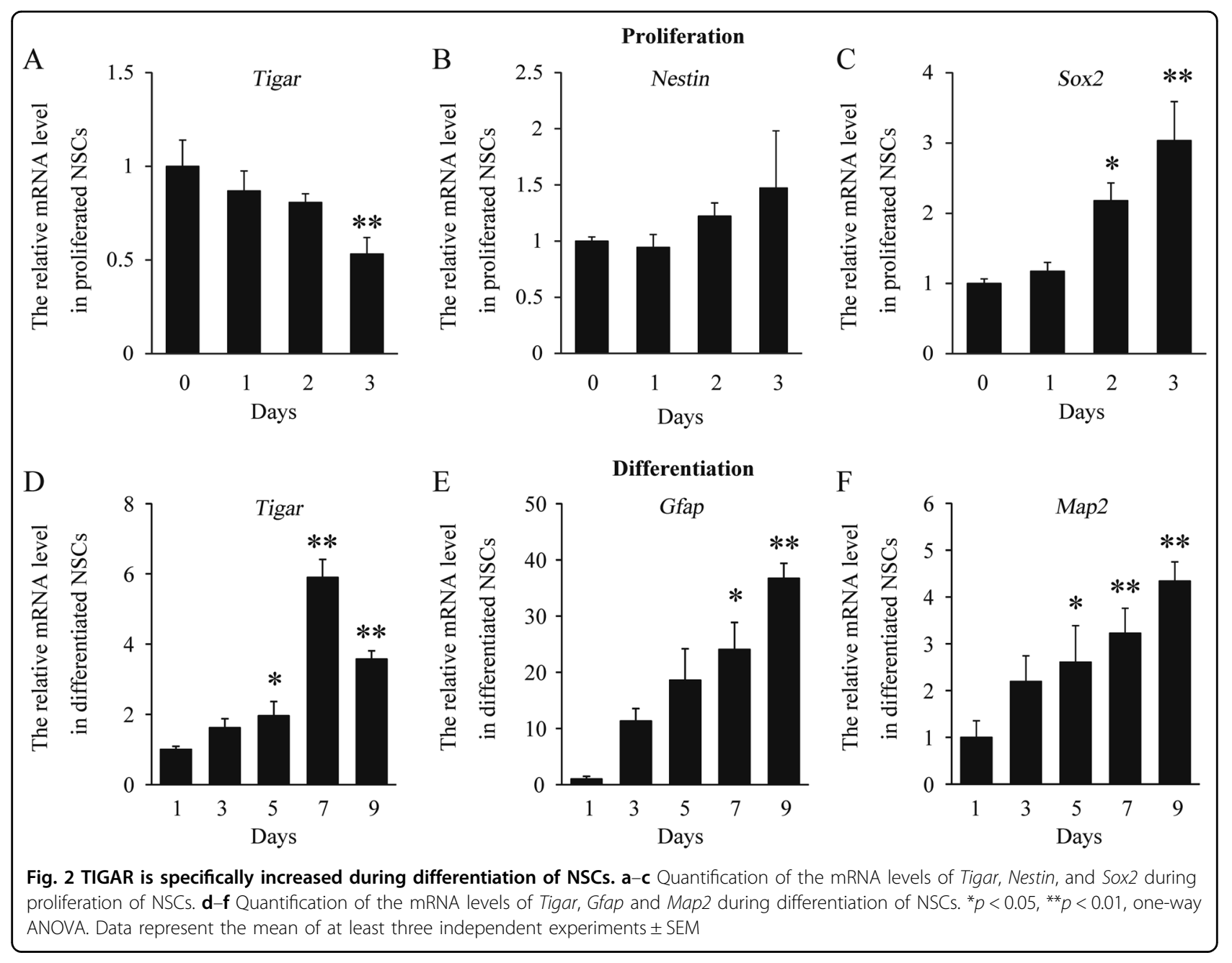

(ACSS2) and citrate-dependent ATP-citrate lyase (ACLY) are two important enzymes that participate in the production of acetyl-CoA for histone acetylation ${ }^{24}$. As shown in Fig. 5j, knockdown of TIGAR significantly decreased the mRNA level of Acly. It is possible that the decrease in mitochondrial activity and mitochondrial acetyl-CoA level may influence the expression of ACLY. Using protocols from a previous study, we generated the Tigar-TM mutant (triple mutant H11A/E102A/H198A) to abolish TIGAR metabolic enzyme activity in fructose bisphosphatase (FBPase-2) ${ }^{6}$. Overexpression of TIGAR-TM mutant lost its effect on glycolysis and OXPHOS (Fig. S1A, B). Importantly, we found that blocking the metabolic enzyme activity of TIGAR abolished its effect on the expression of Acly (Fig. S1C). This suggests that the effect of TIGAR on ACLY expression may be related to the regulation of mitochondrial activity and the level of acetyl-CoA in mitochondria. Overall, we concluded that TIGAR enhanced mitochondrial metabolism, and increased the level of acetyl-CoA during NSC differentiation.
TIGAR regulates NSC differentiation by increasing the level of acetyl-CoA

Acetyl-CoA, a central metabolic intermediate, has been shown to be an important second messenger that regulates histone acetylation ${ }^{23}$. Therefore, we tested for changes in histone acetylation in Lenti-siTigar-treated NSCs. Our data showed that knockdown of TIGAR did not alter the level of H3K18, H3K14 or H3K27 acetylation (Fig. 6a). However, acetylation of H3K9 was significantly decreased after loss of TIGAR (Fig. 6a). As expected, overexpression of TIGAR increased H3K9 acetylation (Fig. 6b).

Evidence has shown that histone acetylation is an important component of epigenetic regulation to activate gene transcription ${ }^{25}$. Therefore, we asked whether H3K9 acetylation can regulate the expression of NSC differentiation-related genes. Our ChIP results showed that the levels of H3K9 acetylation at the promoters of the Ngn1, Neurod1, and Gfap genes were significantly decreased after knockdown of TIGAR in differentiated NSCs (Fig. 6c). Acetyl-CoA can be generated from acetate 


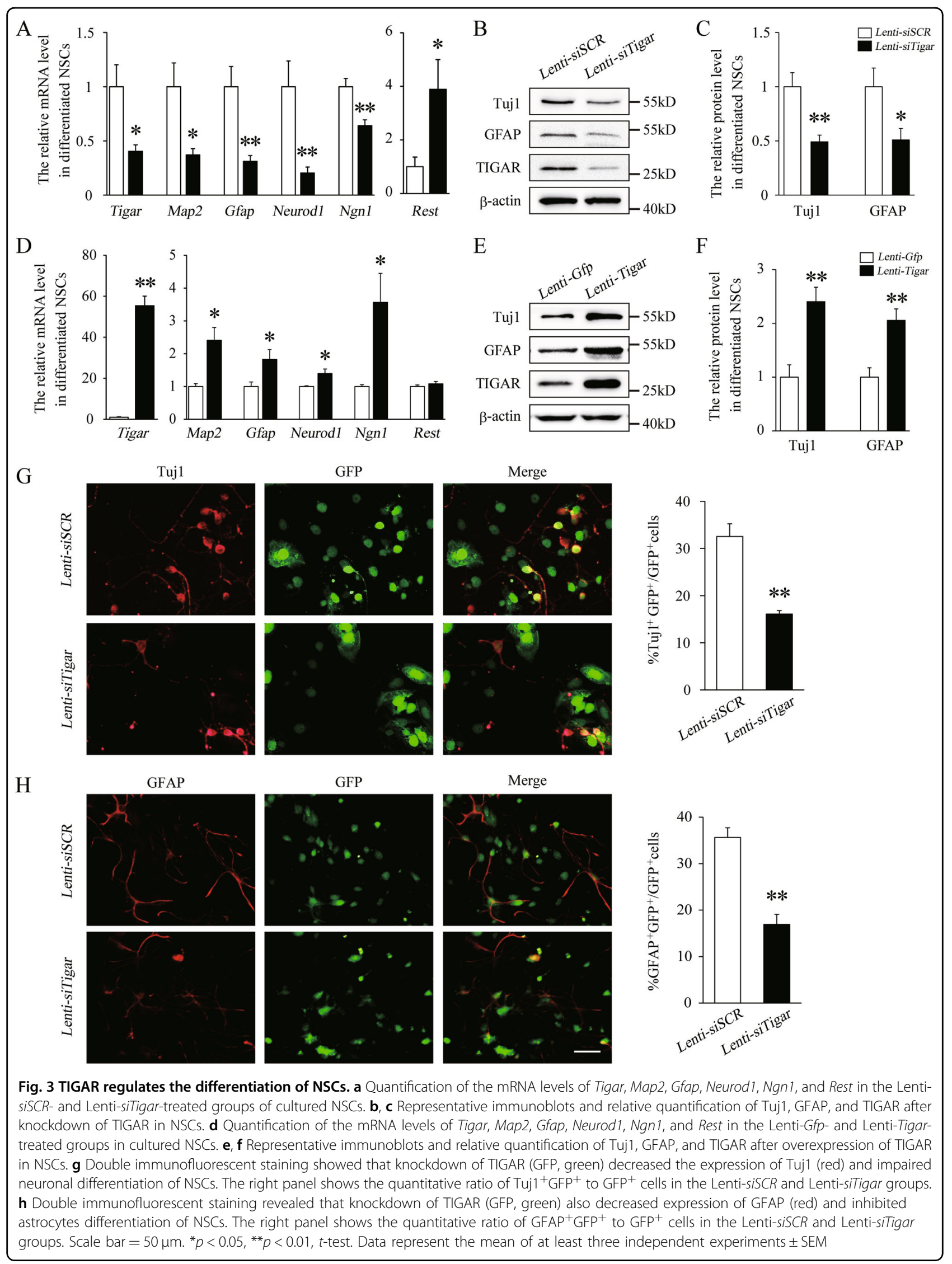



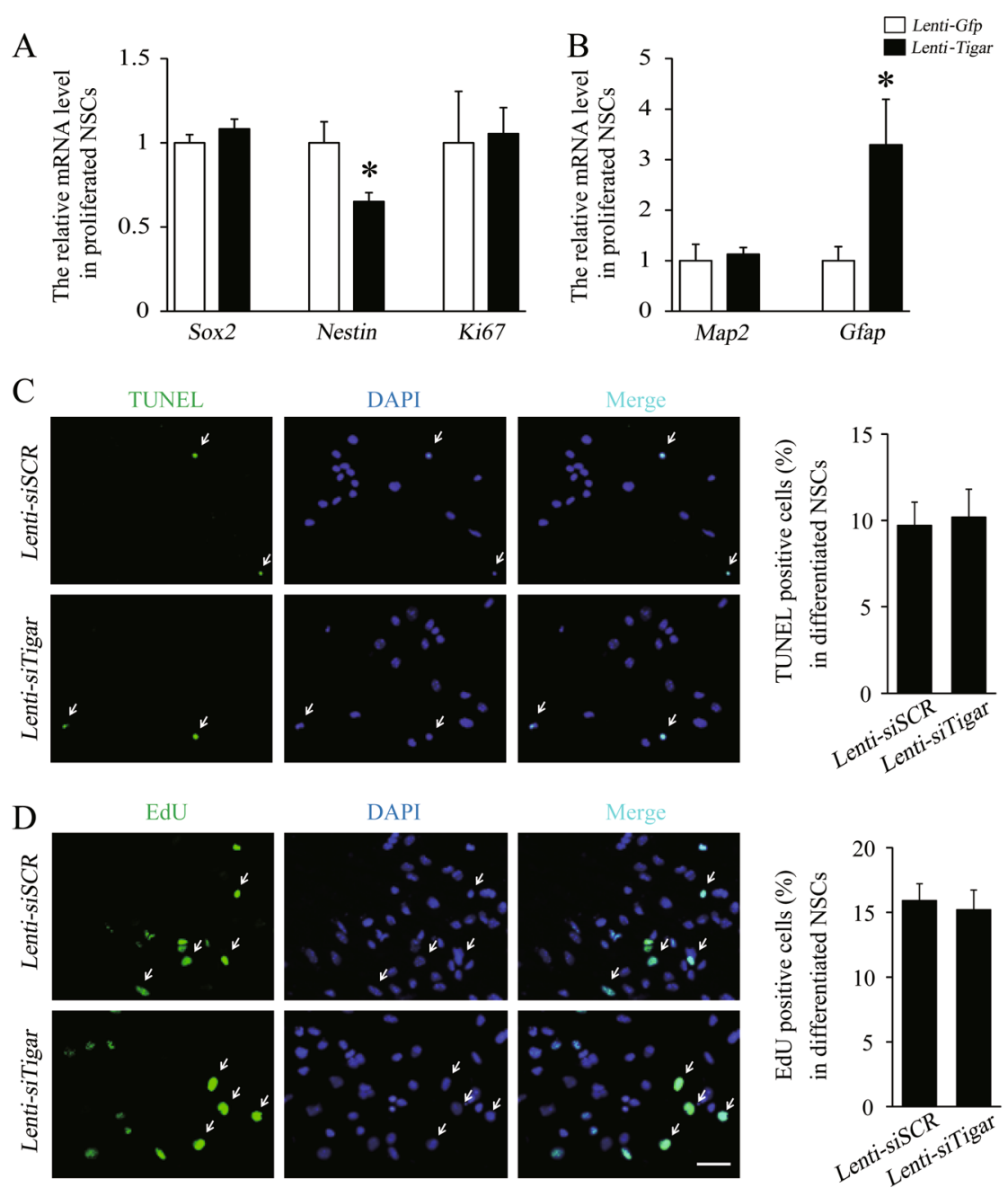

Fig. 4 TIGAR has no effect on the cell survival or proliferation of NSCs. a, b Quantification of the mRNA levels of Sox2, Nestin, Ki67, Map2, and Gfap in the Lenti-Gfp- and Lenti-Tigar-treated groups of proliferated NSCs. c TUNEL staining (green) in cultured NSCs treated with Lenti-siSCR or LentisiTigar during the differentiation stage. The right panel shows the quantification of TUNEL-positive cells in the Lenti-siSCR and Lenti-siTigar groups. Scale bar $=50 \mu \mathrm{m}$. $\mathbf{d}$ During the differentiation stage, there was no significant difference in the number of dividing NSCs in the Lenti-siSCR and LentisiTigar groups. The right panel shows the quantification of EdU-positive cells in the Lenti-siSCR and Lenti-siTigar groups. Scale bar $=50 \mu \mathrm{m} .{ }^{*} p<0.05$, ${ }^{* *} p<0.01, t$-test. Data represent the mean of at least three independent experiments \pm SEM

by ACSS2, which is independent of citrate ${ }^{26}$. As shown in Fig. 6d, supplementation with acetate reversed LentisiTigar-induced decrease of acetyl-CoA level. Importantly, acetate significantly increased the level of H3K9 acetylation and rescued the effect of Lenti-siTigar on the decrease in H3K9 acetylation in NSCs (Fig. 6e). Furthermore, qPCR and western blotting showed that acetate reversed the decreases in MAP2, Tuj1, and GFAP expression caused by knockdown of TIGAR (Fig. 6f, g). Together, our results suggest that TIGAR may regulate NSC differentiation via increases in acetyl-CoA levels and H3K9 acetylation at the promoters of Gfap, Neurod1, and Ngn1.

\section{Discussion}

Metabolic reprogramming from aerobic glycolysis to OXPHOS is a hallmark of neuronal differentiation ${ }^{4,12}$. However, it remains unknown what triggers metabolism transition and how glucose metabolism directs neuronal differentiation. TIGAR, which acts as endogenous inhibitor of glycolysis and is widely distributed in neurons, attracted our interest. The results of this study provide several new insights into the effect of TIGAR on NSC differentiation. First, TIGAR is highly expressed in NSCs and increases as neural differentiation proceeds during embryonic cortex development. In vitro experiments further demonstrated that TIGAR is necessary and 


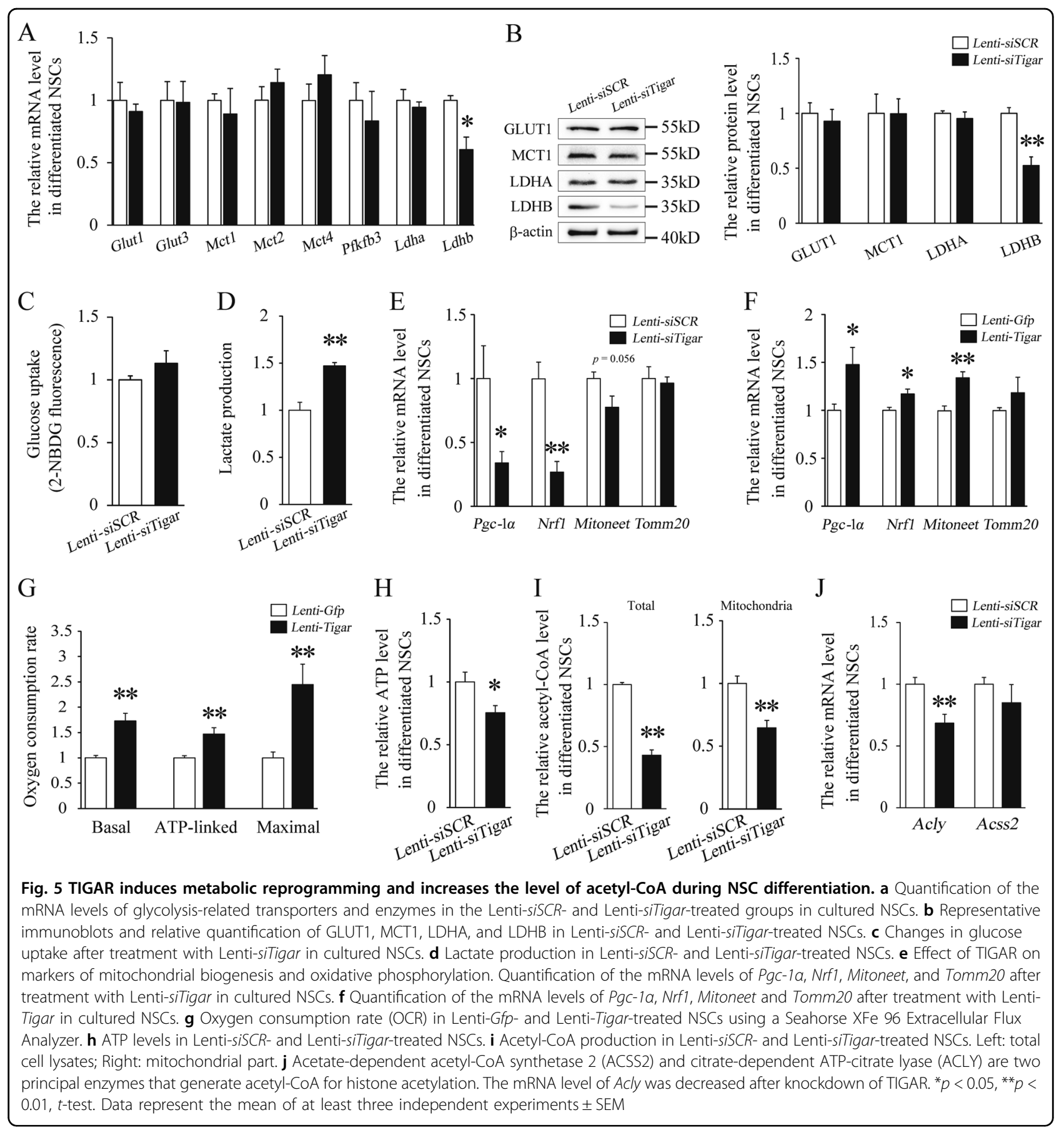

sufficient for NSC differentiation. Second, TIGAR promoted a shift in glucose metabolism to a mitochondrial phenotype and increased the levels of acetyl-CoA and H3K9 acetylation during NSC differentiation. Third, TIGAR might direct NSC differentiation via the upregulation of acetyl-CoA and H3K9 acetylation Fig. 7.

TIGAR is a potential candidate for metabolic regulation and directs neural differentiation. Glycolysis and mitochondrial OXPHOS are two major pathways that provide energy and biosynthetic precursors to cells. The balance between glycolysis and OXPHOS is essential for biological activity in cells. Regulation of metabolic programming is regarded as an important strategy to control cell fate and relieve injury caused by disease. In this study, TIGAR was selected as a potential target through which to regulate metabolism in NSCs. First, we found that the expression of TIGAR in the embryonic cortex was increased at E12.5-E16.5. Furthermore, immunostaining results 


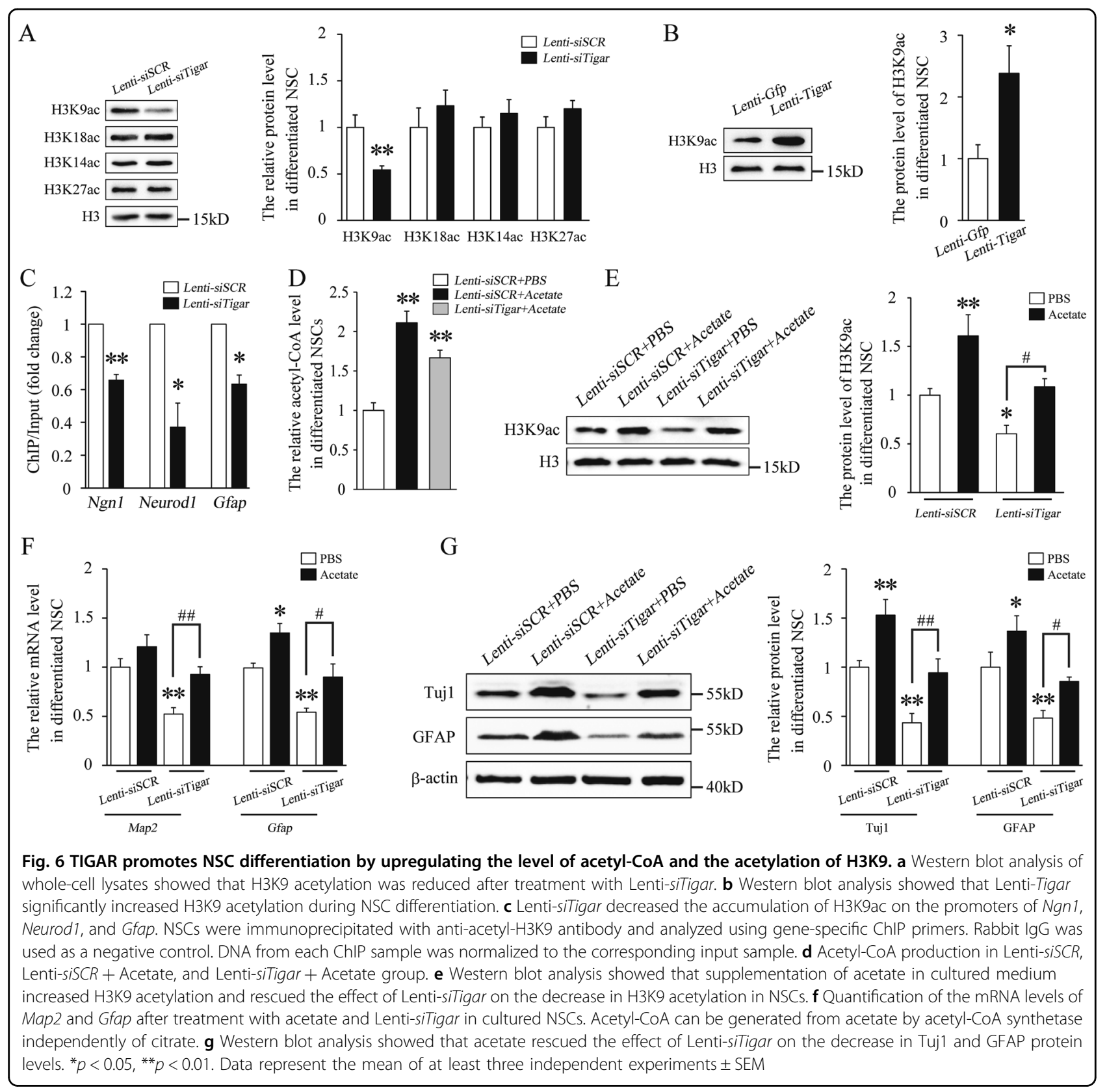

demonstrated that TIGAR was highly expressed in NSCs in the VZ/SVZ and CP at E16.5. It has been reported that, during the development of the mouse neocortex, neurogenesis occurs around E12, peaks around E15 and finishes around birth ${ }^{20}$. Thus, TIGAR has the same expression pattern as the initiation of neural differentiation in the embryonic cortex.

Our in vitro experiments demonstrated that TIGAR is necessary and sufficient for NSC differentiation. Previous studies have reported that the levels of many metabolic enzymes changed dramatically during neural differentiation, including GLUTs, HK2, LDHA, and PKM ${ }^{11,12,27}$.
However, we regard TIGAR as a regulator of glucose metabolism rather than merely a step in the process. TIGAR was initially identified as a p53-inducible gene. Studies have reported that p53 is a key factor in NSCs and can regulate neuronal differentiation at multiple levels ${ }^{28,29}$. In cancer cells, p53 inhibits glycolysis and restricts intracellular reactive oxygen species (ROS) levels through TIGAR and promotes OXPHOS by upregulating cytochrome c oxidase $2(\mathrm{SCO} 2)^{6,30}$. According to our data, TIGAR may act as a very important bridge between p53 and metabolic reprogramming during NSC differentiation. 


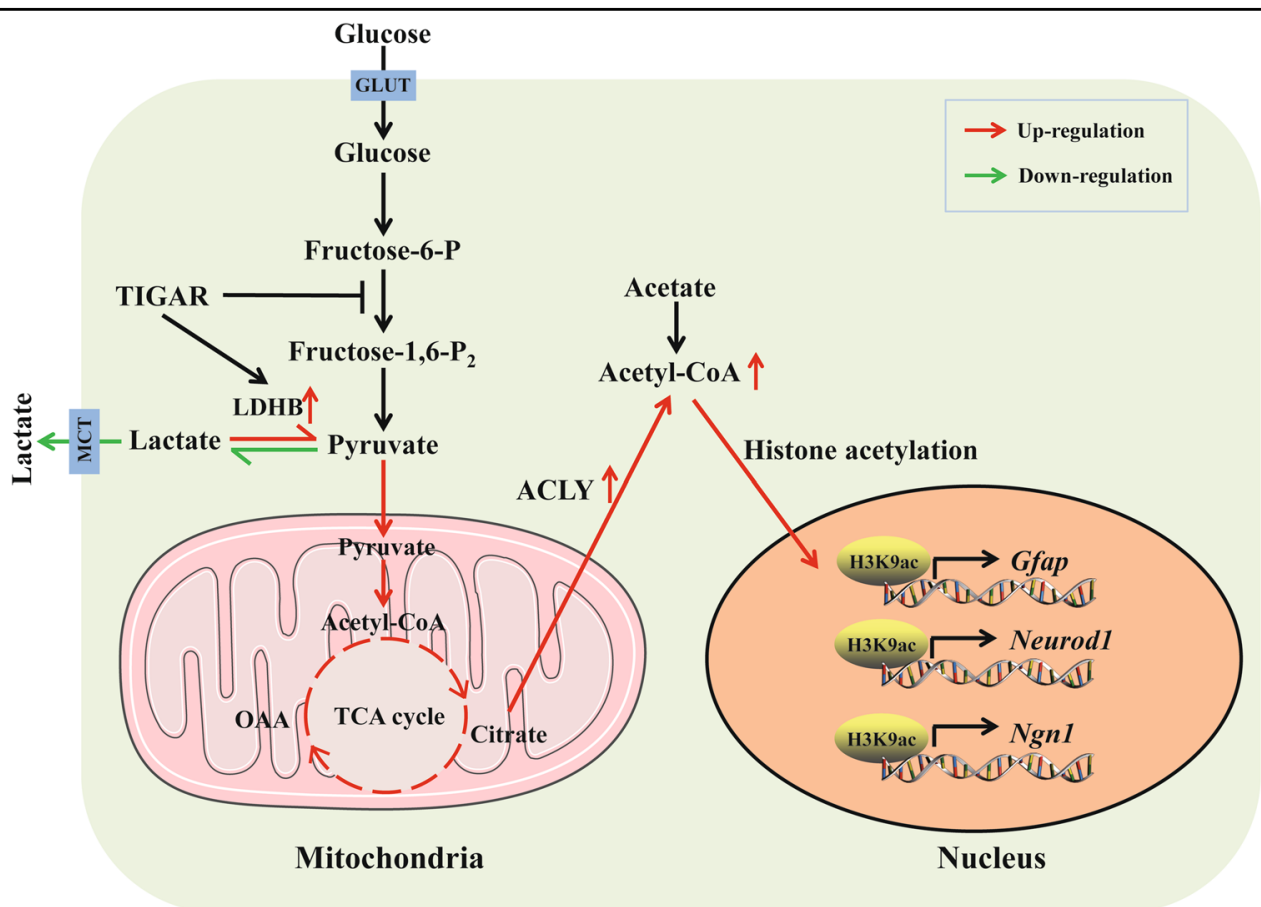

Fig. 7 Schematic representation of TIGAR-induced neural differentiation of NSCs. TIGAR enhanced mitochondrial biogenesis and oxidative phosphorylation by increasing the expression of $\mathrm{Ldhb}, \mathrm{Pgc}-1 \mathrm{a}$, Nrfl, and Mitoneet, which triggers increases in acetyl-CoA and H3K9 acetylation. The upregulation of H3K9 acetylation resulted in the transcriptional activation of Ngn1, Neurod1, and Gfap during NSC differentiation

Furthermore, TIGAR accelerated glucose metabolism to a mitochondrial phenotype. Our data showed that TIGAR had no effect on the mRNA levels of Gluts, Mcts, Pfkfb3, or Ldha or on the uptake of glucose. However, knockdown of TIGAR decreased the expression of LDHB and enhanced lactate production during neural differentiation. LDHB converts lactate into pyruvate to be further oxidized in mitochondria ${ }^{31}$. This suggests that TIGAR may enhance OXPHOS by increasing the level of pyruvate via LDHB. Evidence has shown that overexpression of TIGAR in carcinoma cells increases the expression of PGC-1 $\alpha$, NRF1, MitoNEET, and Tomm20, which are important markers of mitochondrial biogenesis and OXPHOS $^{32}$. Similarly, our data showed that TIGAR increased the expression of PGC- $1 \alpha$, NRF1, and MitoNEET, and enhanced oxygen consumption rate and ATP generation in differentiated NSCs. This suggested that TIGAR enforced a mitochondrial oxidative phosphorylation phenotype during NSC differentiation. In addition, acetyl-CoA, a key metabolite during OXPHOS, influences many cellular processes, including energy metabolism, autophagy, and cell proliferation and differentiation ${ }^{23,33}$. In this study, we found that knockdown of TIGAR reduced both whole cell and mitochondrial acetyl-CoA. Evidence has shown that acetyl-CoA can be generated from mitochondria-derived citrate by the cytosolic enzyme ACLY ${ }^{23}$. Our data showed that knockdown of
TIGAR decreased the expression of ACLY. Citrate is an important intermediate in the tricarboxylic acid (TCA) cycle, and its abundance increases as mitochondrial OXPHOS is enhanced ${ }^{34,35}$. Citrate can be exported to the cytosol via the dicarboxylate antiporter solute carrier family 25, member 1 (SLC25A1), and converted into acetyl-CoA and oxaloacetate by $\mathrm{ACLY}^{36}$. In this study, blocking the metabolic enzyme activity of TIGAR abolished its effect on glycolysis and OXPHOS, and the expression of Acly. Therefore, we hypothesized that inhibition of mitochondrial OXPHOS and mitochondrial acetyl-CoA generation by knockdown of TIGAR might influence acetyl-CoA derived citric acid and the expression of ACLY.

Finally, TIGAR regulated neural differentiation through an epigenetic mechanism. Mounting evidences have shown that the production of acetyl-CoA is closely linked to the regulation of histone acetylation ${ }^{23,37}$. However, the effect of acetyl-CoA in NSCs is not clear. We demonstrated that TIGAR specifically regulated H3K9 acetylation during NSC differentiation. Furthermore, ChIP experiments showed that TIGAR enhanced the levels of H3K9 acetylation at the Ngn1, Neurod1, and Gfap promoters. These results demonstrated that TIGAR regulated the expression of genes related to NSC differentiation by increasing H3K9 acetylation. Acetate, a precursor of acetyl-CoA, rescued the decreases in H3K9 
acetylation and MAP2 and GFAP expression resulting from knockdown of TIGAR. Thus, we demonstrated that TIGAR epigenetically directed NSC differentiation.

In summary, our work reveals a novel mechanism of TIGAR-regulated NSC differentiation. Future developmental clinical experiments may identify TIGAR as a potential target for the treatment of neurological diseases.

\section{Acknowledgements}

This work was supported by funding from the National Natural Science Foundation of China (NO. 81571093 and 81771219), and the Natural Science Foundation of Shandong Province (NO. 2018GSF118076).

\section{Author contributions}

W.Z. designed and performed the majority of the laboratory work, the analysis of the data and the writing of the manuscript; T.Z., J.D. and G.J. were involved in collection and assembly of data. X.L., S.J., W.T., and X.W. contributed to the data analysis and interpretation. A.H. was involved in data analysis and interpretation, conception and design, manuscript writing, financial support, and final approval of the manuscript.

\section{Conflict of interest}

The authors declare that they have no conflict of interest.

\section{Publisher's note}

Springer Nature remains neutral with regard to jurisdictional claims in published maps and institutional affiliations.

Supplementary Information accompanies this paper at (https://doi.org/ 10.1038/s41419-019-1434-3).

Received: 9 August 2018 Revised: 28 November 2018 Accepted: 10 December 2018

Published online: 27 February 2019

\section{References}

1. Temple, S. The development of neural stem cells. Nature 414, 112 (2001).

2. Goldman, StevenA. Stem and progenitor cell-based therapy of the central nervous system: hopes, hype, and wishful thinking. Cell Stem Cell 18, 174-188 (2016).

3. Kameda, T., Imamura, T. \& Nakashima, K. Epigenetic regulation of neural stem cell differentiation towards spinal cord regeneration. Cell Tissue Res. 371, 189-199 (2018).

4. Kim, D.-Y., Rhee, I. \& Paik, J. Metabolic circuits in neural stem cells. Cell. Mol. Life Sci. 71, 4221-4241 (2014).

5. Lopes, C. \& Rego, A. C. Revisiting mitochondrial function and metabolism in pluripotent stem cells: where do we stand in neurological diseases? Mol. Neurobiol. 54, 1858-1873 (2016).

6. Bensaad, K. et al. TIGAR, a p53-inducible regulator of glycolysis and apoptosis. Cell 126, 107-120 (2006).

7. Ko, Y.-H. et al. TP53-inducible Glycolysis and Apoptosis Regulator (TIGAR) metabolically reprograms carcinoma and stromal cells in breast cancer. J. Biol. Chem. 291, 26291-26303 (2016).

8. Li, M. et al. A TIGAR-regulated metabolic pathway is critical for protection of brain ischemia. J. Neurosci. 34, 7458-7471 (2014).

9. Bélanger, M., Allaman, I., Magistretti \& Pierre, J. Brain energy metabolism: focus on astrocyte-neuron metabolic cooperation. Cell. Metab. 14, 724-738 (2011).
10. Benarroch, E. E. Brain glucose transporters: implications for neurologic disease. Neurology 82, 1374-1379 (2014).

11. M., C. K., William, S. C. \& Anna, C. L. Neural stem/progenitor cells display a low requirement for oxidative metabolism independent of hypoxia inducible factor-1alpha expression. J. Neurochem. 125, 420-429 (2013).

12. Zheng, $X$. et al. Metabolic reprogramming during neuronal differentiation from aerobic glycolysis to neuronal oxidative phosphorylation. eLife 5, e13374 (2016).

13. Chen, $X$. et al. Protein palmitoylation regulates neural stem cell differentiation by modulation of EID1 activity. Mol. Neurobiol. 53, 5722-5736 (2016).

14. Jie, F. et al. Melatonin promotes proliferation and differentiation of neural stem cells subjected to hypoxia in vitro. J. Pineal Res. 51, 104-112 (2011).

15. Kong, L. et al. Direct neuronal glucose uptake is required for contextual fear acquisition in the dorsal hippocampus. Front. Mol. Neurosci. 10, 388 (2017).

16. Shi, G. \& McQuibban, G. A. The mitochondrial rhomboid protease PARL is regulated by PDK2 to Integrate mitochondrial quality control and metabolism. Cell Rep. 18, 1458-1472 (2017).

17. Pashkovskaia, N., Gey, U. \& Rödel, G. Mitochondrial ROS direct the differentiation of murine pluripotent P19cells. Stem Cell Res. 30, 180-191 (2018).

18. Ferreira, J. M., Burnett, A. L. \& Rameau, G. A. Activity-dependent regulation of surface glucose transporter-3. J. Neurosci. 31, 1991-1999 (2011).

19. Flavahan, W. A. et al. Brain tumor initiating cells adapt to restricted nutrition through preferential glucose uptake. Nat. Neurosci. 16, 1373-1382 (2013).

20. Qian, X. et al. Timing of CNS cell generation: a programmed sequence of neuron and glial cell production from isolated murine cortical stem cells. Neuron 28, 69-80 (2000).

21. Ross, S. E., Greenberg, M. E. \& Stiles, C. D. Basic helix-loop-helix factors in cortical development. Neuron 39, 13-25 (2003).

22. Sun, Y.-M. et al. Distinct profiles of REST interactions with its target genes at different stages of neuronal development. Mol. Biol. Cell. 16, 5630-5638 (2005)

23. Pietrocola, F. et al. Acetyl Coenzyme A: A central metabolite and second messenger. Cell. Metab. 21, 805-821 (2015).

24. Mews, P. et al. Acetyl-CoA synthetase regulates histone acetylation and hippocampal memory. Nature 546, 381-386 (2017).

25. Qiao, Y., Wang, R., Yang, X., Tang, K. \& Jing, N. Dual roles of histone H3 lysine 9 acetylation in human embryonic stem cell pluripotency and neural differentiation. J. Biol. Chem. 290, 2508-2520 (2015).

26. Peng, M. et al. Aerobic glycolysis promotes $\mathrm{T}$ helper 1 cell differentiation through an epigenetic mechanism. Science 354, 481-484 (2016).

27. Maurer, M. H., Geomor, H. K., Bürgers, H. F., Schelshorn, D. W. \& Kuschinsky, W. Adult neural stem cells express glucose transporters GLUT1 and GLUT3 and regulate GLUT3 expression. FEBS Lett. 580, 4430-4434 (2006).

28. Meletis, K. et al. p53 suppresses the self-renewal of adult neural stem cells. Development 133, 363-369 (2006).

29. Forsberg, $K$. et al. The tumor suppressor p53 fine-tunes reactive oxygen species levels and neurogenesis via PI3 kinase signaling. J. Neurosci. 33 14318-14330 (2013)

30. Matoba, S. et al. p53 regulates mitochondrial respiration. Science $\mathbf{3 1 2}$ 1650-1653 (2006)

31. Le, A. et al. Inhibition of lactate dehydrogenase $A$ induces oxidative stress and inhibits tumor progression. Proc. Natl Acad. Sci. USA 107, 2037-2042 (2010).

32. Ko, Y.-H. et al. TIGAR metabolically reprograms carcinoma and stromal cells in breast cancer. J. Biol. Chem. 291, 26291-26303 (2016).

33. Moussaieff, A. et al. Glycolysis-mediated changes in acetyl-CoA and histone acetylation control the early differentiation of embryonic stem cells. Cell. Metab. 21, 392-402 (2015).

34. Peng, M. et al. Aerobic glycolysis promotes $T$ helper 1 cell differentiation through an epigenetic mechanism. Science 354, 481-484 (2016).

35. Mondal, S. et al. HSulf-1 deficiency dictates a metabolic reprograming of glycolysis and TCA cycle in ovarian cancer. Oncotarget 6, 33705-33719 (2015).

36. Zaidi, N., Swinnen, J. V. \& Smans, K. ATP-citrate lyase: a key player in cancer metabolism. Cancer Res. 72, 3709-3714 (2012)

37. Wellen, K. E. et al. ATP-citrate lyase links cellular metabolism to histone acetylation. Science 324, 1076-1080 (2009). 